\title{
Evaluating the Negative or Valuing the Positive? Neural Mechanisms Supporting Feedback-Based Learning across Development
}

\author{
Anna C. K. van Duijvenvoorde, ${ }^{1,2,3}$ Kiki Zanolie, ${ }^{1,3,4}$ Serge A. R. B. Rombouts, ${ }^{1,3,5}$ Maartje E. J. Raijmakers, ${ }^{2}$ and \\ Eveline A. Crone ${ }^{1,3}$ \\ ${ }^{1}$ Leiden University Institute for Psychological Research, Leiden University, 2333 AK Leiden, The Netherlands, ${ }^{2}$ Department of Developmental Psychology, \\ University of Amsterdam, 1018 WB Amsterdam, The Netherlands, ${ }^{3}$ Leiden Institute for Brain and Cognition, Leiden University, 2300 RC Leiden, The \\ Netherlands, ${ }^{4}$ Department of Psychology, Erasmus University Rotterdam, 3000 DR Rotterdam, The Netherlands, and ${ }^{5}$ Department of Radiology, Leiden \\ University Medical Center, 2333 ZA Leiden, The Netherlands
}

\begin{abstract}
How children learn from positive and negative performance feedback lies at the foundation of successful learning and is therefore of great importance for educational practice. In this study, we used functional magnetic resonance imaging (fMRI) to examine the neural developmental changes related to feedback-based learning when performing a rule search and application task. Behavioral results from three age groups (8-9,11-13, and 18-25 years of age) demonstrated that, compared with adults, 8- to 9-year-old children performed disproportionally more inaccurately after receiving negative feedback relative to positive feedback. Additionally, imaging data pointed toward a qualitative difference in how children and adults use performance feedback. That is, dorsolateral prefrontal cortex and superior parietal cortex were more active after negative feedback for adults, but after positive feedback for children (8 -9 years of age). For 11 - to 13-year-olds, these regions did not show differential feedback sensitivity, suggesting that the transition occurs around this age. Presupplementary motor area/anterior cingulate cortex, in contrast, was more active after negative feedback in both 11- to 13-year-olds and adults, but not 8- to 9-year-olds. Together, the current data show that cognitive control areas are differentially engaged during feedbackbased learning across development. Adults engage these regions after signals of response adjustment (i.e., negative feedback). Young children engage these regions after signals of response continuation (i.e., positive feedback). The neural activation patterns found in 11to 13-year-olds indicate a transition around this age toward an increased influence of negative feedback on performance adjustment. This is the first developmental fMRI study to compare qualitative changes in brain activation during feedback learning across distinct stages of development.
\end{abstract}

Key words: development; fMRI; prefrontal cortex; cognitive control; learning; feedback

\section{Introduction}

One of the key foundations for successful learning, such as in school situations, is feedback-based learning, which refers to our ability to use performance feedback in subsequent behavior. Both positive and negative feedback are important for improving performance, signaling either a continuation or an adjustment of the current behavior. When presented with negative feedback, adults adjust behavior more successfully than 8- to 11-year-old children (Crone et al., 2004, 2008; Huizinga et al., 2006). In contrast, positive feedback can function as a reinforcer to continue current behavior. For example, developmental studies show that chil-

Received Jan. 6, 2008; revised July 2, 2008; accepted Aug. 5, 2008.

This work was supported by Netherlands Organisation for Scientific Research-VIDI grants (S.A.R.B.R., E.A.C) and by the European Commission Framework6 Project 516542 (New and Emerging Science and Technologies) (M.E.J.R.). We thank Dietsje Jolles and Wouter van den Bos for their help with data analysis.

Correspondence should be addressed to Dr. Eveline A. Crone, Department of Developmental Psychology, Leiden University Institute for Psychological Research, Leiden University, Wassenaarseweg 52, 2333 AK Leiden, The Netherlands. E-mail: ecrone@fsw.leidenuniv.nl.

DOI:10.1523/JNEUROSCI.1485-08.2008

Copyright $\odot 2008$ Society for Neuroscience $\quad$ 0270-6474/08/289495-09\$15.00/0 dren's cognitive performance improves on a rule shift task when positive stimuli are used (Qu and Zelazo, 2007). However, the neural mechanisms that support these behavioral differences are currently unknown.

Medial prefrontal cortex (PFC), including the anterior cingulate cortex (ACC) and supplementary motor area (SMA)/preSMA (Rushworth et al., 2004, 2007), and dorsolateral PFC (DLPFC) are often found to be more active after the presentation of negative performance feedback in adults (Mars et al., 2005; Zanolie et al., 2008a). Pre-SMA/ACC is thought to signal response conflict and to predict activation in DLPFC, a region that is considered to be important for subsequent performance adjustment (Kerns et al., 2004). Previous developmental functional magnetic resonance imaging (fMRI) studies demonstrated that pre-SMA/ACC and DLPFC undergo developmental changes both functionally (Crone et al., 2006b) and structurally (Gogtay et al., 2004).

In addition to medial PFC and DLPFC, superior and inferior parietal cortex are also often implicated in cognitive and behavioral regulation, such as stimulus conflict detection (Liston et al., 


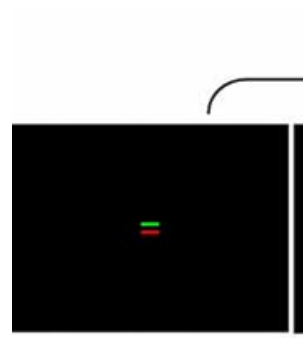

$500 \mathrm{msec}$

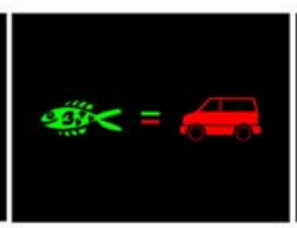

$2000 \mathrm{msec}$

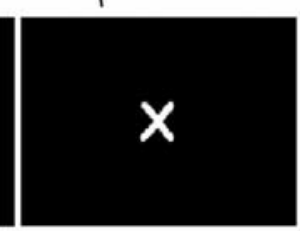

$1000 \mathrm{msec}$

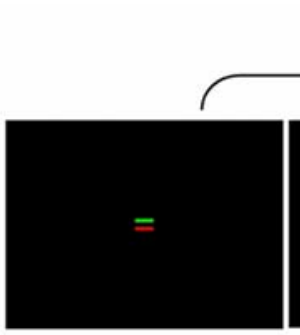

$500 \mathrm{msec}$

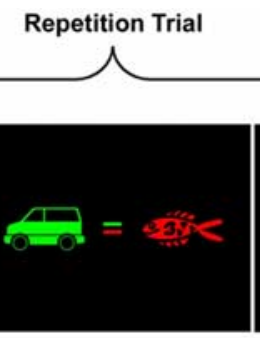

$2000 \mathrm{msec}$

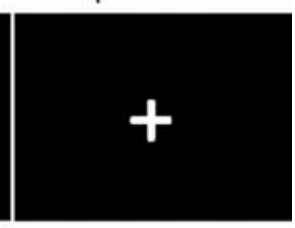

$1000 \mathrm{msec}$
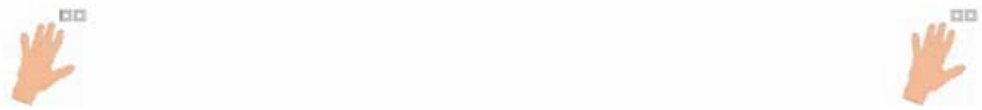

Figure 1. Task design: trials were paired into guess and repetition trials. A cue indicated the dimension to select (shape or color) and was followed by the stimulus presentation. A response was followed by positive $(+)$ or negative $(x)$ feedback, after which the sequence repeated during the repetition trial. Trials were separated by intertrial intervals (not shown) of $2-8 \mathrm{~s}$, during which a central fixation cross was shown.

2006), response selection (Bunge et al., 2002), and feedback processing (Crone et al., 2008). A previous developmental fMRI study that focused on negative feedback processing demonstrated developmental differences in engagement of not only DLPFC and pre-SMA/ACC, but also superior parietal cortex (Crone et al., 2008), which is consistent with previous reports demonstrating structural changes in parietal cortex across development (Gogtay et al., 2004).

Finally, the caudate, with its interconnections to the prefrontal cortex, has been implicated in the learning of stimulus-response associations and is found to be sensitive to positive rather than to negative feedback (Toni et al., 2002; Seger and Cincotta, 2006; Cincotta and Seger, 2007). Structural imaging studies indicate that the caudate's developmental trajectory is similar to that of frontal and parietal regions (Giedd, 2008).

The goal of this study was to investigate the neural developmental changes related to the ability to use positive and negative performance feedback for subsequent behavioral adjustment. We developed a child-friendly task in which participants adjusted performance based on feedback by responding to either the color or shape of the stimuli. Despite the developmental changes in feedback-based learning and cognitive control generally between ages 8 and 12 (Cepeda et al., 2001; Crone et al., 2004), previous fMRI studies have collapsed across 8 - to 12 -year-olds. We focused on the changes within this age range by collecting behavioral and fMRI measurements in three age groups: 8- to 9-yearolds, 11- to 13-year-olds, and 18- to 25-year-olds. Based on previous research, we examined feedback sensitivity in DLPFC, pre-SMA/ACC, parietal cortex, and caudate. We expected increased pre-SMA/ACC, DLPFC, and superior parietal cortex activation in 18- to 25-year-olds after negative feedback and caudate activation after positive feedback. This will be the first study to examine how these regions contribute to feedback-based rule learning after negative relative to positive feedback across childhood.

\section{Materials and Methods}

Participants. Fifty-five healthy volunteers were included in the study: 18 adults ( 11 female, 7 male; ages $18-25 ; M=22.3$ ), 19 early adolescents ( 8 female, 11 male; ages $11-13 ; M=11.7$ ), and 18 children ( 8 female, 10 male; ages $8-9 ; M=8.6$ ). Participants were paid volunteers, recruited through local advertisements or through a university course credits system. All participants were right handed and reported normal or corrected-to-normal vision and an absence of neurological or psychiatric impairments. For children aged 8-9 and 11-13 years, the Child Behavior
Checklist was filled out by the parents to secure an absence of psychiatric conditions. All participants had total scores below the clinical range. Participants gave informed consent for the study; for minors, the primary caretaker gave informed consent for participation. All procedures were approved by the Leiden University Department of Psychology and the medical ethical committee of the Leiden University Medical Center. Two early adolescents were excluded from further analyses because of poor task performance in the experimental phase, in which performance was $<55 \%$ correct for either the positive or negative feedback condition. Three additional participants (one early adolescent and two children) were excluded because of excessive head movement (in total $>3 \mathrm{~mm}$ of translation in any direction). Therefore, our final sample consisted of 18 adults, 16 adolescents, and 16 children.

Standard intelligence scores were obtained from each participant using the Raven's Progressive Matrices test (Raven, 1941). Estimated mean intelligence quotients were 117 for 8- to 9-year-olds, 118 for 11- to 13year-olds, and 123 for 18- to 25-year-olds and did not differ significantly between age groups $(p=0.11)$. The results from the adults have been reported previously to confirm the involvement of DLPFC and preSMA/ACC in feedback processing (Zanolie et al., 2008b). In this previous study, we reported the effects of intradimensional and extradimensional switches, but this was not the focus of the current study.

Task. The task was a rule selection and application task in which participants were instructed to use positive and negative feedback to select and apply a simple rule (i.e., one-dimensional rule) for target stimulus selection (Zanolie et al., 2008b). The relevant rule set was cued by a centrally presented equals sign and could be based either on color (colored equals sign) or on shape (white equals sign) of the stimuli. Stimuli consisted of different pairs of nameable pictures that all differed on two dimensions: color and shape (e.g., a red fish and a green car). These paired stimuli were presented on the left and right sides of the cue. Both their positions and colors were interchangeable within each pair, such that both values of each stimulus dimension were present.

Stimuli were always presented in pairs of trials, in which participants had to select the correct picture (left or right located stimulus picture of the pair) by means of a middle or index finger button press from the left hand (Fig. 1). In the first trial (referred to below as the guess trial), participants had been cued the rule set (color rules or shape rules), but did not yet know the correct response [expected $p$ (correct) $=0.5$ ]. For example, a participant cued to apply the "color" rule on the guess trial might arbitrarily select the red item as being correct. Participants' responses generated a visually presented positive $(+)$ or negative $(x)$ feedback stimulus in the center of the screen, which indicated a correct or incorrect choice, respectively. Feedback was followed by the second trial (referred to as the repetition trial), in which the rule set and the stimulus pair were repeated, although the colors and/or shapes of the pictures could be switched. Participants had to choose the correct item based on feedback they had received on the guess trial. Thus, if participants cor- 
rectly used feedback after the guess trials, they could perform at $100 \%$ accuracy on repetition trials. For every consecutive guess trial, a different stimulus pair was presented (with different shape and color values), and the rule set (color rules, shape rules) was either repeated or changed.

Trials had the following structure: a centrally located cue was presented for $500 \mathrm{~ms}$, followed by a combined stimulus presentation and response window for $2 \mathrm{~s}$, after which feedback was presented for $1 \mathrm{~s}$. Timing was identical for guess and repetition trials. Intertrial intervals were jittered in $\sim 25 \%$ of the trials. This jitter varied exponentially from $2,4,6$, and $8 \mathrm{~s}$, based on an optimalization program [optseq2; see http:// surfer.nmr.mgh.harvard.edu/optseq/, developed by Dale (1999)]. During intertrial intervals, a central fixation cross was shown.

Participants were familiarized with the scanner environment on the day of the fMRI session through the use of a mock scanner, which simulated the sounds and environment of a real MRI scanner. Immediately before scanning, children and adults practiced the behavioral task in a quiet laboratory to ensure proficiency on the task. All participants were informed that their task in the experiment would be to make simple decisions about pairs of pictures by means of a button press, and they were instructed to respond as accurately as possible. During training outside the scanner, participants received 15 practice trials of each rule type, first a color and then a shape block, followed by one mixed block of 30 trials. In the fMRI session, participants completed four experimental blocks, all consisting of 100 trials each. Technical malfunctions or excessive head movement rendered one block of trials unusable for one participant in the 11- to 13-year-old and one in the 8- to 9-year-old age group.

MRI procedure. AMRI data were acquired with a standard whole-head coil using a 3-Tesla Philips Achieva scanner in the Leiden University Medical Center. T2*-weighted echoplanar images (EPIs) were obtained during four functional runs of 232 volumes each, of which the first two were discarded to allow for equilibration of T1 saturation effects. Each volume covered the whole brain (38 slices of thickness $2.75 \mathrm{~mm}$; field of view, $220 \mathrm{~mm} ; 80 \times 80$ matrix; in-plane resolution, $2.75 \mathrm{~mm}$ ) and was acquired every $2200 \mathrm{~ms}$ (echo time $=30 \mathrm{~ms}$, ascending interleaved acquisition). A high-resolution T1-weighted anatomical scan was obtained from each participant after the functional runs. Visual stimuli were projected onto a screen located at the head of the scanner bore and viewed by participants by means of a mirror mounted to the head coil assembly. In accordance with Leiden University Medical Center policy, all anatomical scans were reviewed and cleared by the radiology department after each scan. No anomalous findings were reported.

fMRI data analysis. Data were preprocessed using SPM2 (Wellcome Department of Cognitive Neurology). Images were corrected for differences in timing of slice acquisition, followed by rigid body motion correction. Functional volumes were spatially normalized to EPI templates. The normalization algorithm used a 12-parameter affine transformation together with a nonlinear transformation involving cosine basis functions and resampled the volumes to $3 \mathrm{~mm}$ cubic voxels. Templates were based on the MNI305 stereotaxic space (Cocosco et al., 1997), an approximation of Talairach space (Talairach and Tourneaux, 1988). Functional volumes were spatially smoothed with an $8 \mathrm{~mm}$ full-width at halfmaximum isotropic Gaussian kernel. Statistical analyses were performed on individual subjects' data using the general linear model in SPM2. The fMRI time series data were modeled by a series of events convolved with a canonical hemodynamic response function (HRF). The feedback stimulus of each trial (guess and repetition trials) was modeled as a zeroduration event. Trials on which participants did not respond within the $2 \mathrm{~s}$ time window or trials that were followed by an incorrect trial on the repetition trial were modeled separately and were not included in the pairwise contrasts of interest. The modeled events were used as covariates in a general linear model, along with a basic set of cosine functions that high-pass filtered the data, and a covariate for session effects. The leastsquares parameter estimates of height of the best-fitting canonical HRF for each condition were used in pairwise contrasts. The resulting contrast images, computed on a subject-by-subject basis, were submitted to group analyses. At the group level, contrasts between conditions were computed by performing one-tailed $t$ tests on these images, treating subjects as a random effect. Task-related responses were considered signifi-

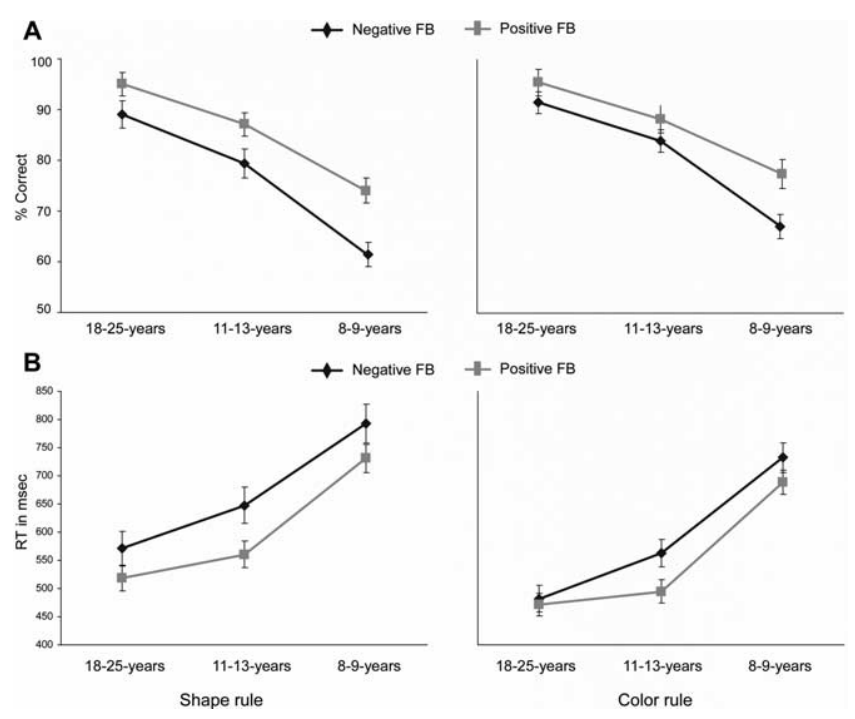

Figure 2. $\quad \boldsymbol{A}, \boldsymbol{B}$, Performance on repetition trials after receiving negative and positive feedback $(\mathrm{FB})$ indicated by percentages correct $(\boldsymbol{A})$ and reaction time $(\boldsymbol{B})$. Results are presented separately for rule type (shape and color), with SEs plotted.

cant if they consisted of at least 10 contiguous voxels that exceeded an uncorrected threshold of $p<0.001$. We performed region of interest (ROI) analyses to further test for age $\times$ condition effects.

ROI analyses were performed with the Marsbar toolbox in SPM2 (Brett et al., 2002) (http://marsbar.sourceforge.net/). ROIs that spanned several functional brain regions were subdivided by sequentially masking the functional ROI with each of several anatomical Marsbar ROIs. The contrast used to generate functional ROIs was based on the general contrast all feedback stimuli $>$ fixation, $p<0.005$ (uncorrected) across all participants. For all ROI analyses, effects were considered significant at an $\alpha$ of 0.0125 , based on Bonferroni correction for multiple comparisons ( $p=0.05 / 4$ ROIs), unless reported otherwise. For each ROI, the center of mass is reported.

\section{Results}

\section{Performance}

First, to investigate the general behavioral effect of feedback presentation between age groups, guess trial response accuracy (by design of the task at 50\% accuracy) was used to establish a performance baseline. The difference score in accuracy between guess trials and repetition trials was analyzed with a between-agegroup (8-9, 11-13, and 18-25) ANOVA. Results showed that, as expected, the amount of accuracy improvement was greater for young adults $(M=43 \%)$ than for 11 - to 13 -year-olds $(M=35 \%)$ and 8 - to 9-year-olds $(M=20 \%)$ (main effect of age group, $F_{(2,47)}$ $=20.43 ; p<0.001$ ) (Fig. $2 A$ ). Post hoc tests confirmed that differences were significant for all age comparisons (all $p$ values $<$ $0.05)$.

Second, to examine accuracy differences on repetition trials after positive and negative feedback, an age group (8-9, 11-13, and $18-25) \times$ rule type (color and shape) $\times$ feedback (negative and positive) ANOVA was performed. This analysis was performed on repetition trials only because accuracy for guess trials, by design of the task, was at chance level for all age groups. As expected, general accuracy on repetition trials was higher for young adults $(M=93 \%)$ than for 11 - to 13 -year-olds $(M=85 \%)$ and 8- to 9-year-olds $(M=70 \%)$ (main effect of age group, $F_{(2,47)}$ $=29.34 ; p<0.001)$. Post hoc tests confirmed that differences were significant for all age comparisons (all $p$ values $<0.05$ ).

Further, accuracy on repetition trials was higher after receiving positive feedback on the previous guess trial $(M=87 \%)$ than 
after negative feedback $(M=79 \%)$ (main effect of feedback, $\left.F_{(1,47)}=75.05 ; p<0.001\right)$. The decrease in performance on repetition trials after receiving negative feedback was larger for children (age group $\times$ feedback interaction, $F_{(2,47)}=3.37 ; p<0.05$ ) (Fig. 2A). Post hoc comparisons confirmed that the difference between positive and negative feedback accuracy was larger for 8to 9-year-olds than for young adults, and 11- to 13-year-olds performed at an intermediate level. Finally, sorting according to shape $(M=81 \%)$ was slightly but significantly more effortful than sorting according to color rules $(M=84 \%)$ (main effect of rule type, $\left.F_{(1,47)}=20.01 ; p<0.001\right)$. This effect did not differ significantly between age groups $(p>0.1)$.

By subdividing the task into four blocks of 50 trials (total 200 trials), we examined also whether there were differential learning patterns during the task between age groups. The age group $\times$ feedback $X$ task block ANOVA showed no difference in overall accuracy between age groups across task blocks (age group $\times$ task block interaction, $p=0.11$ ), nor a difference in accuracy after positive or negative feedback across task blocks (age group $\times$ feedback $\times$ task block interaction, $p=0.86$ ). Thus, there were no differential learning patterns across task blocks for positive relative to negative feedback learning between age groups.

Finally, an age group $(8-9,11-13$, and $18-25) \times$ rule type (color and shape) $\times$ feedback (negative and positive) ANOVA was performed to examine reaction time (RT) differences on repetition trials, which showed comparable results with accuracy analyses. Overall, mean RTs were faster for young adults $(M=$ $511 ; \mathrm{SD}=98)$ and 11 - to 13 -year-olds $(M=579 ; \mathrm{SD}=98)$ than for 8- to 9-year-olds $(M=744 ; \mathrm{SD}=86)$ (main effect of age group, $\left.F_{(2,47)}=29.25 ; p<0.001\right)$. Furthermore, results showed that participants were faster on repetition trials after receiving positive feedback $(M=585 ; \mathrm{SD}=120)$ than after receiving negative feedback $(M=638$; SD $=145)$ (main effect of feedback, $\left.F_{(1,47)}=30.17 ; p<0.001\right)$. Finally, sorting according to shape resulted in slower RTs $(M=643 ; \mathrm{SD}=134)$ than sorting according to color rules $(M=580 ; \mathrm{SD}=128)$ (main effect of rule type, $\left.F_{(1,47)}=62.36 ; p<0.001\right)($ Fig. $2 B$ ).

To summarize, the behavioral results show that young adults performed more accurately in general and showed a larger behavioral improvement after feedback presentation than 11- to 13year-olds, who in turn performed more accurately and showed a larger behavioral improvement than 8- to 9-year-olds. Furthermore, these results indicate that positive and negative feedback differentially influenced subsequent performance adjustment across age groups. Negative feedback in general resulted in lower performance accuracy and slower RTs on subsequent repetition trials than positive feedback. However, this decrease in accuracy after negative feedback was larger for the younger participants. Thus, when feedback is negative, i.e., requires a behavioral adjustment, that adjustment is considered more effortful for the youngest age group. In addition, all participants were less accurate and slower to apply the shape sorting than the color sorting rules.

\section{Whole-brain comparisons}

The brain analyses focused specifically on feedback after guess trials, because this feedback was informative for performance on the next trial. Only those trials on which participants performed accurately on the repetition trials were included in the analysis. The number of guess trials included was 140 for 8- to 9-year-olds, 160 for 11 - to 13 -year-olds, and 180 for 18 - to 25 -year-olds. For each age group, the amount of guess trials included consisted of half positive feedback, half negative feedback.

The regions that were active for the negative $>$ positive and positive $>$ negative contrasts are presented in Figure $3(p<$ 0.001 , at least 10 contiguous voxels). The coordinates are reported in supplemental Table 1 (available at www.jneurosci.org as supplemental material) for the comparison negative $>$ positive feedback and supplemental Table 2 (available at www. jneurosci.org as supplemental material) for the comparison positive $>$ negative feedback for each age group separately.

The comparison negative feedback $>$ positive feedback for young adults resulted in activation in pre-SMA/ACC [Brodmann area (BA) 6], left DLPFC (BA 9), right anterior prefrontal cortex (BA 10), and bilateral inferior and superior parietal cortex (BA $7 / 40)$. The positive feedback $>$ negative feedback comparison resulted in activation in medial frontal gyrus (BA 10/11), precuneus (BA 31), and parahippocampal gyrus (BA 35, 36).

The comparison negative $>$ positive feedback for 11- to 13year-olds resulted in activation in left pre-SMA/ACC (BA 6) and right superior lateral PFC (BA 8). The reversed positive $>$ negative contrast resulted in activation in medial frontal cortex (BA 10).

The comparison negative $>$ positive feedback for 8 - to 9 -yearolds did not show any significant activation. The reversed positive $>$ negative contrast resulted in activation in regions that were sensitive to the reversed contrast in the older age groups, including pre-SMA/ACC (BA 6), superior lateral PFC (BA 8), and left superior parietal areas (BA 7).

Together, the whole-brain contrasts suggest that age groups were differentially sensitive to positive and negative feedback that was informative for subsequent performance continuation and adjustment.

To test whether the youngest (8- to 9-year-olds) and oldest (18- to 25-year-olds) age groups had statistically different brain responses to positive and negative feedback, we performed two sample $t$ tests (age group $\times$ feedback) on whole-brain positive versus negative feedback contrasts to compare the youngest age group (8- to 9-year-olds) and young adults (18- to 25-year-olds) ( $p<0.001$, uncorrected, at least 10 contiguous voxels). Activation maps and the table of activations are presented in the supplemental material.

The comparison negative feedback $>$ positive feedback for 18 - to 25-year-olds $>8$ - to 9-year-olds, and the positive feedback $>$ negative feedback for 8 - to 9-year-olds $>18$ - to 25-yearolds, resulted in activation in bilateral pre-SMA/ACC (BA 6), right DLPFC (BA 9/46), bilateral anterior prefrontal cortex (BA $10)$, left inferior and superior parietal cortex (BA 7/40), caudate, and claustrum. The comparison positive feedback $>$ negative feedback for 18 - to 25 -year-olds $>8$ - to 9-year-olds, and the comparison negative feedback $>$ positive feedback for 8 - to 9 -year-olds $>18$ - to 25 -year-olds, did not show any significant activation.

Additionally, we performed ROI analyses to examine the agerelated feedback effects for different brain areas in more detail and to determine how the differences in feedback sensitivity between age groups were affected by rule types.

\section{ROI analyses}

We performed ROI analyses for four a priori defined regions, which were selected based on the unbiased all feedback events $>$ fixation contrast across participants: $\operatorname{DLPFC}(-44,19,40)$, pre$\operatorname{SMA} / A C C(-3,16,51)$, superior parietal cortex $(-25,-67,70)$, and caudate $(17,6,14)$. One additional ROI analysis was performed for anterior lateral PFC $(-40,51,2)$, which was selected empirically, based on its consistent activation patterns in the whole-brain contrasts. The results of this region are discussed in 

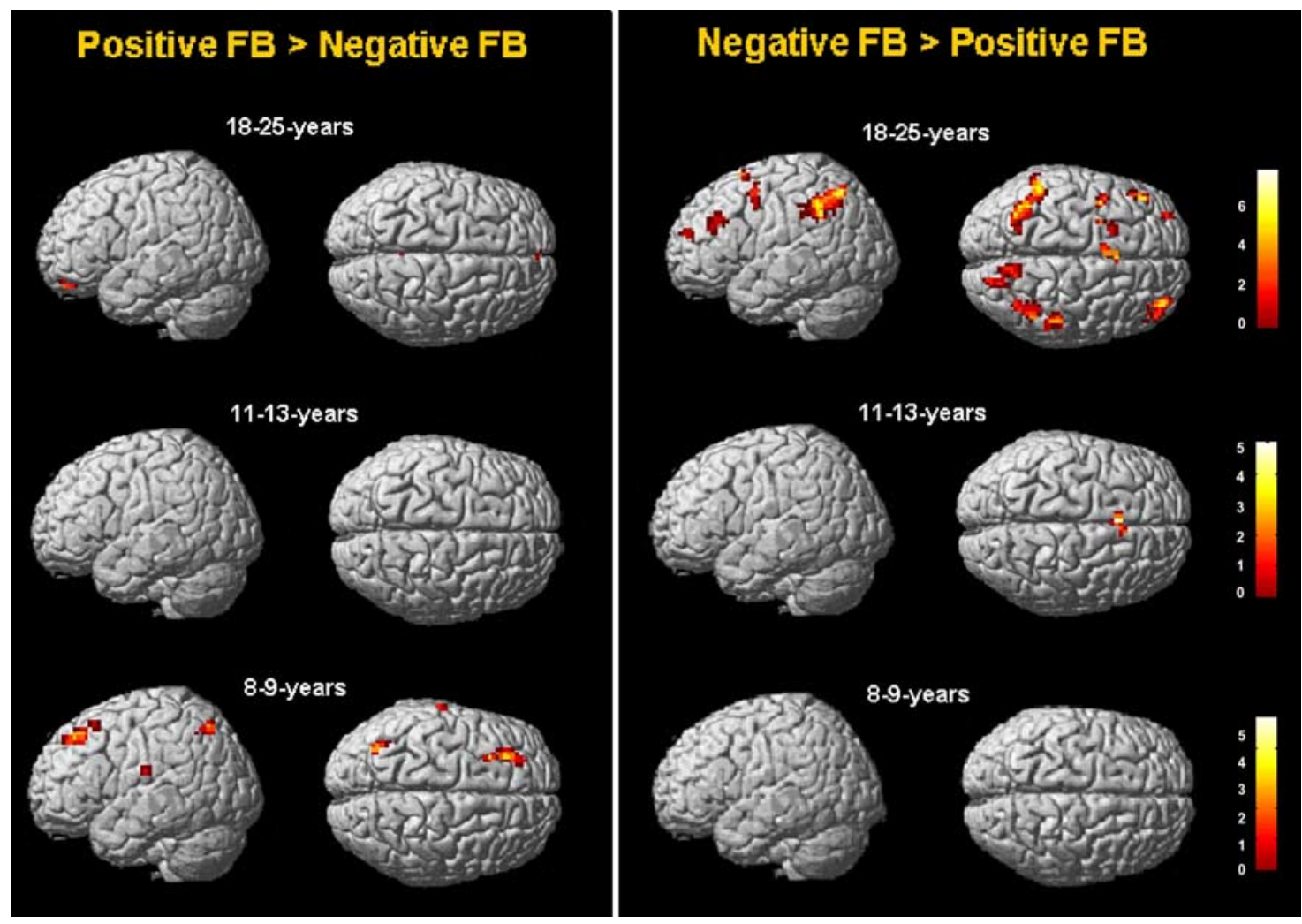

Figure 3. Feedback-locked whole-brain contrasts are displayed for 18-to 25-year-olds, 11- to 13-year-olds, and 8- to 9-year-olds, showing effects of negative feedback (FB) $>$ positive FB at $p<$ 0.001 (uncorrected) versus effects of positive FB $>$ negative FB at $p<0.001$ (uncorrected). Coordinates are reported in supplemental Tables 1 and 2 (available at www.jneurosci.org as supplemental material).

the supplemental results (available at www.jneurosci.org as supplemental material).

We focused on left lateralized regions because these showed the strongest effects (except for caudate), but we additionally compared these effects with the right lateralized ROIs, because of bilateral activation patterns seen in the whole-brain contrasts at more lenient thresholds. As in the whole-brain comparisons, the analyses focused only on feedback after guess trials and were only included when subsequent repetition trials were performed correctly. The data are organized by feedback (positive and negative) and rule type (color and shape). The age $\times$ feedback interactions for pre-SMA/ACC, left DLPFC, left superior parietal cortex, and right caudate are presented in Figure 4.

\section{Pre-SMA/ACC}

The age group $\times$ feedback $\times$ rule type ANOVA for pre-SMA/ ACC resulted in the expected age group $\times$ feedback interaction $\left(F_{(2,47)}=5.93 ; p<0.005\right)$. Post hoc comparisons for each age group demonstrated that activation in pre-SMA/ACC was larger after negative feedback than after positive feedback for young adults $\left(F_{(1,17)}=12.44 ; p<0.005\right)$ and for 11 - to 13 -year-olds $\left(F_{(1,15)}=15.32 ; p<0.001\right)$. For the 8- to 9-year-olds, however, pre-SMA/ACC did not show a differential activation pattern for positive versus negative feedback ( $p=0.55$ ) (Fig. 4).

\section{DLPFC}

The same ANOVA for left DLPFC also resulted in the expected age group $\times$ feedback interaction $\left(F_{(2,47)}=8.73 ; p<0.001\right)$. Post hoc comparisons for each age group demonstrated that activation in left DLPFC was larger after negative feedback than after positive feedback for young adults $\left(F_{(1,17)}=11.73 ; p<0.005\right)$. In contrast, 8 - to 9 -year-olds showed the reversed pattern, in which activation was larger after positive performance feedback than after negative performance feedback $\left(F_{(1,15)}=4.48 ; p=0.05\right)$ (Fig. 4). Eleven- to thirteen-year-olds did not show a differential activation pattern for positive versus negative feedback ( $p=$ $0.80)$.

A similar analysis for right DLPFC resulted in the same age group $\times$ feedback interaction $\left(F_{(2,47)}=7.53 ; p<0.001\right)$. A comparison between left and right DLPFC did not result in a significant interaction with region $(p=0.75)$.

\section{Superior parietal cortex}

The ANOVA for left superior parietal cortex also resulted in an age group $\times$ feedback interaction $\left(F_{(2,47)}=13.3 ; p<0.001\right)$. Post hoc comparisons for each age group demonstrated that activation in left superior parietal cortex was larger after negative feedback than after positive feedback for young adults $\left(F_{(1,15)}=12.48 ; p<\right.$ $0.005)$. In contrast, 8 - to 9 -year-olds showed the reversed pattern, in which activation was larger after positive performance feedback than after negative feedback $\left(F_{(1,15)}=9.7 ; p<0.005\right)$. The 11 - to 13 -year-olds did not show a differential activation pattern for positive versus negative feedback ( $p=0.97$ ) (Fig. 4).

A similar analysis for right superior parietal cortex resulted in the same age group $\times$ feedback interaction $\left(F_{(2,47)}=6.18 ; p<\right.$ 0.005 ) with similar results for post hoc comparisons. A comparison between left and right superior parietal cortex did not result in a significant interaction with region for this effect $(p=0.56)$.

\section{Caudate}

Finally, the same analysis for left caudate did not show a significant effect of feedback $(p=0.11)$. In contrast, the ANOVA for right caudate showed a main effect for feedback $\left(F_{(1,47)}=5.11\right.$; $p=0.03)$, where positive feedback resulted in higher activation 

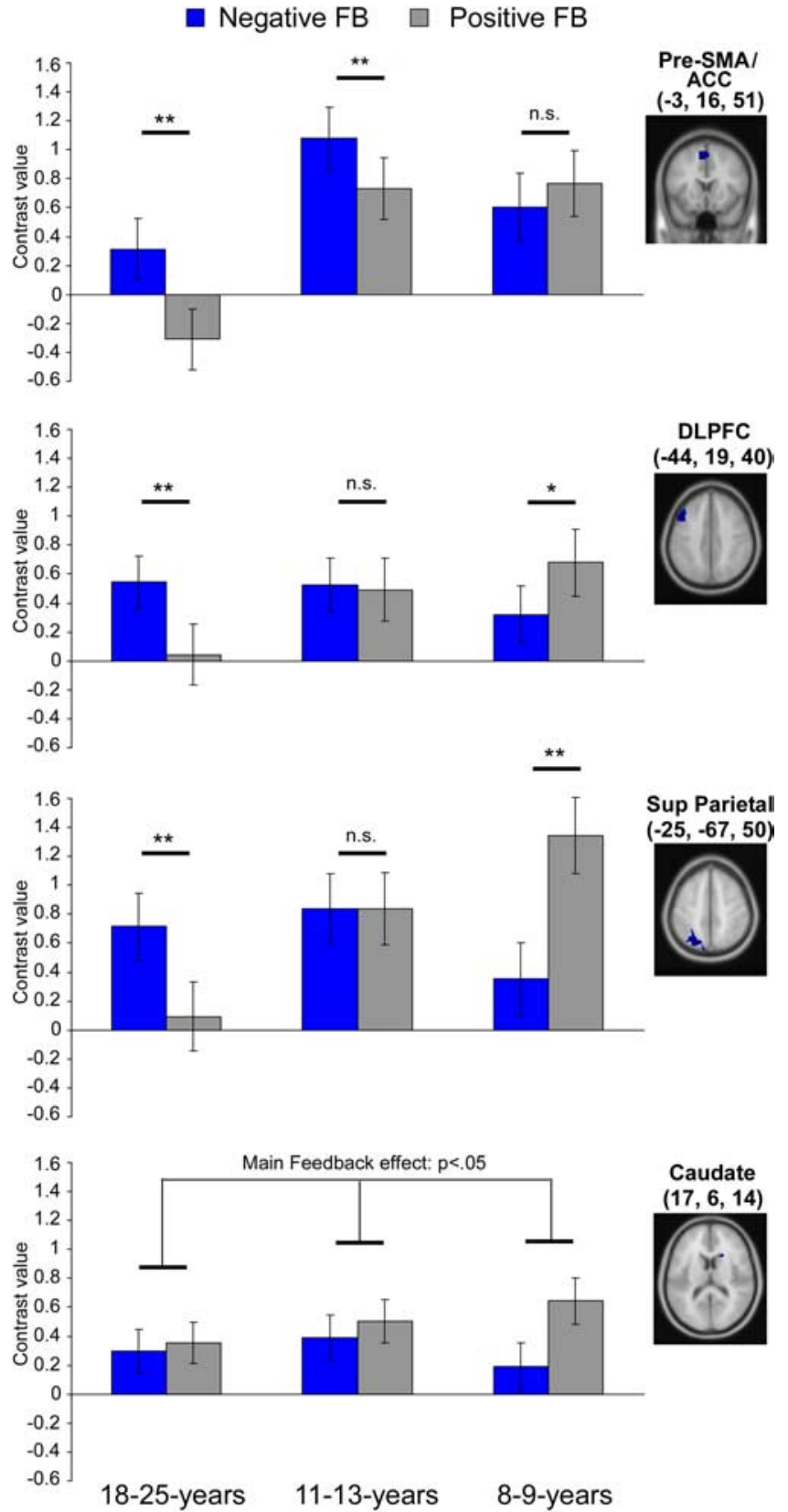

Figure 4. ROl activation values for pre-SMA/ACC, left DLPFC, left superior (Sup) parietal cortex, and right caudate for each age group. For the first three regions, an age group (18-25, $11-13$, and $8-9) \times$ feedback (negative and positive) interaction was present (all $p$ values $<$ 0.005 ) (post hoc analyses indicating significant differences between positive and negative feedback, ${ }^{*} p$ values $<0.05 ;{ }^{* *} p$ values $\left.<0.005\right)$. SEs are plotted.

levels than negative feedback. This effect did not differ across age groups ( $p=0.27$ ) (Fig. 4).

To summarize, the ROI analyses resulted in three main effects: (1) DLPFC and superior parietal cortex were more active after negative than positive feedback in adults, but after positive than negative feedback in 8- to 9-year-olds. Eleven- to thirteen-yearolds did not reveal a differential sensitivity toward negative and positive feedback in these regions. (2) Pre-SMA/ACC was more active after negative than positive feedback in adults and 11- to 13-year-olds, whereas 8- to 9-year-olds did not demonstrate feedback sensitivity in this region. (3) The caudate was more active after positive feedback than after negative feedback, and this effect did not differ between age groups.
Three additional analyses were conducted to account for several challenges when comparing participants of different age groups: (1) correction for possible carryover effects of previous negative feedback trials, (2) correction for number of trials included in the analysis, and (3) correction for head movement differences.

Carryover effects of previous trials

We conducted an additional analysis to test whether the observed differences in brain activation were related to possible carryover effects of previous feedback, given that 8- to 9-year-old children received more negative feedback in general. We performed a sequential analysis, in which guess trials followed by negative feedback were recoded into two types: those for which the previous repetition trial was correct and those for which the previous repetition trial was incorrect. Similar recoding was done for guess trials followed by positive feedback. For these recoded variables, the feedback on each trial was modeled as a zero-duration event in the fMRI analyses. Comparisons were conducted for the youngest age group separately, given that this was the age group with the highest prevalence of incorrect repetition trials, and across age groups. Results showed that the previous trial did not modulate the feedback effects or the age $\times$ feedback interactions in all previous reported ROIs (all $p$ values including effects of previous feedback were NS). Also, for the youngest age group separately, no interactions of previous feedback $\times$ feedback (negative vs positive) were observed in the ROIs for pre-SMA/ACC, DLPFC, superior parietal cortex, and caudate (all $p$ values $>$ $0.10)$.

\section{Matching for number of observations}

We conducted a second additional analysis to test whether the observed differences in brain activation were related to differences in the number of observations between age groups. We equated the average number of correct trials included in the fMRI analyses across age groups. For both adults and 11- to 13-yearolds, we selected a random subset of $70 \%$ of the correct trials, making the average percentage of correct trials $71 \%$ for young adults, $72 \%$ for 11 - to 13 -year-olds, and $70 \%$ for 8 - to 9 -yearolds. For this subset of trials, the feedback on all trials was modeled as a covariate of no interest in the fMRI analyses. All feedback $\times$ age group effects reported earlier remained significant for pre-SMA/ACC $\left(F_{(2,47)}=3.6 ; p<0.05\right)$, bilateral DLPFC (left, $F_{(2,47)}=5.1, p<0.02$; right, $\left.F_{(2,47)}=5.2, p<0.01\right)$, and bilateral superior parietal cortex (left, $F_{(2,47)}=11.6, p<0.001$; right, $\left.F_{(2,47)}=5.86, p<0.01\right)$. However, the previous feedback main effect in right caudate was nonsignificant $(p=0.11)$.

\section{Controlling for head motion differences}

To investigate possible head movement differences between age groups, average amount of head movement for each participant was submitted to an age group ANOVA, by averaging the absolute value of translation in $x-y-z$ directions per participants per trial. Results showed a significant effect of age group on average head movement values $\left(F_{(2,47)}=8.8 ; p<0.01\right)$, in which the 8 - to 9 -year-olds moved significantly more than the adults $(p<$ 0.001 ). Movement parameters from 11- to 13-year-olds did not differ significantly from either adults or 8 - to 9-year-olds $(p>$ 0.1 and $p>0.05$, respectively). To control for the head movement differences between age groups, realignment parameters were included in the design matrix, and the same analyses were performed as described above. Results from ROI analyses showed that all feedback $\times$ age group effects reported earlier remained significant for pre-SMA/ACC $\left(F_{(2,47)}=4.4 ; p<0.02\right)$, bilateral 
DLPFC (left, $F_{(2,47)}=7.2, p<0.01$; right, $F_{(2,47)}=5.7, p<0.01$ ), and bilateral superior parietal cortex (left, $F_{(2,47)}=12.3, p<$ 0.001 ; right, $\left.F_{(2,47)}=6.0, p<0.02\right)$. The feedback main effect in right caudate was at trend level $(p=0.078)$.

Together, these additional analyses demonstrate that the age group differences in activation patterns of pre-SMA/ACC, bilateral DLPFC, and bilateral superior parietal cortex could not be accounted for by differences in negative or positive feedback presentation on repetition trials, by different numbers of correct trials being included in the fMRI analyses, or by differences in amount of head movement across age groups.

\section{Correlations}

Finally, we performed correlation analyses for activation in brain areas and behavioral performance within and across age groups, but no significant brain-behavior correlations were found.

\section{Discussion}

The goal of this study was to examine the neural developmental changes related to the ability to use positive and negative performance feedback for subsequent behavioral adjustment in a rule application task. Consistent with previous behavioral studies, all participants performed more accurately and faster on trials that followed positive relative to negative feedback, but 8- to 9-yearold children had more difficulty in learning from negative feedback than adults, resulting in lower accuracy on repetition trials for negative relative to positive feedback trials (Crone et al., 2004; Schmittmann et al., 2006). Brain imaging data yielded three main results: (1) with age, there was a shift in recruitment of DLPFC and superior parietal cortex after positive and negative performance feedback; (2) there were separable developmental trajectories for involvement of DLPFC/superior parietal cortex and pre-SMA/ACC; and (3) the caudate was more active after positive feedback, but this effect was the same for all age groups. The discussion is organized along the lines of these findings.

\section{Sensitivity to positive and negative feedback across development}

Our analysis of sensitivity to positive and negative feedback demonstrated important changes in neural recruitment between ages $8-9,11-13$, and young adults. Whereas young adults showed more activation in DLPFC and superior parietal cortex after negative performance feedback than after positive feedback, this difference was not seen for 11- to 13-year-olds and was reversed for 8 - to 9-year-olds. The adult findings are consistent with previous studies, demonstrating negative feedback-related sensitivity in DLPFC for feedback that is important for subsequent behavioral adjustment (Kerns et al., 2004; Zanolie et al., 2008a). This result confirms its role in the implementation of goal-directed and controlled behavior (Miller and Cohen, 2001). Superior parietal cortex showed a pattern of activation that was similar to the activation pattern seen in DLPFC. Previous imaging research supports these similarities and emphasizes the interplay between prefrontal and parietal cortices in the implementation of cognitive control (Bunge et al., 2002; Brass et al., 2005) and feedback processing (Crone et al., 2008).

Previous developmental studies have suggested immature activation patterns for children in DLPFC and superior parietal cortex related to other cognitive functions, such as working memory (Klingberg et al., 2002; Crone et al., 2006a). In these studies, it was suggested that these regions are not yet accessible to young children because of structural immaturity. The current results indicate that immaturity may also be related to differential sensitivity to the informative value of feedback presentation, or to strategy differences, because children aged 8-9 already recruited prefrontal and parietal regions involved in goal-directed behavior when they received positive feedback. Cognitive developmental studies show that the complexity of strategies for conditional reasoning increases significantly between ages 8 and 13 and is related specifically to working memory capacity (Barrouillet and Lecas, 1999). Possibly, the processing of negative feedback required an additional inference (e.g., if not car, then chair on the next trial), which was more demanding in terms of working memory recruitment than processing positive feedback (e.g., if car is correct, then car again on the next trial). Thus, children may use working memory capacity more successfully after positive feedback than after negative feedback.

Although 11- to 13-year-olds did not recruit DLPFC and superior parietal cortex differentially after positive and negative feedback, they showed more activation in pre-SMA/ACC after negative feedback. In adult studies, pre-SMA/ACC was shown to be sensitive to a negative feedback signal, which indicates that outcomes are worse than anticipated (Holroyd and Coles, 2002) or which signals a potential bad outcome (Brown and Braver, 2005), especially in situations in which the number of response alternatives is large (Walton et al., 2004). Children aged 11-13 showed a pattern of feedback sensitivity in pre-SMA/ACC similar to that in adults, whereas children aged 8-9 did not demonstrate this sensitivity. This finding suggests that the sensitivity of preSMA/ACC to outcome signals that indicate behavioral adjustment (Ullsperger and von Cramon, 2004) develops between ages $8-9$ and $11-13$ years.

\section{Separate developmental trajectories for DLPFC, superior parietal cortex, and pre-SMA/ACC}

The current findings indicate that pre-SMA/ACC matures earlier than DLPFC and superior parietal cortex, suggesting that these regions may have separate contributions to feedback processing. In particular, DLPFC and superior parietal cortex are thought to be sensitive to the informative value of the feedback, because these areas respond to negative feedback in adults, but to positive feedback in 8- to 9-year-olds. Pre-SMA/ACC is thought to be sensitive to conflict and the need for a behavioral change (in the form of presentation of negative feedback) and exhibits a mature pattern of feedback response by age 11-13. These findings are consistent with previous studies, which have demonstrated developmental changes between 8 and 12 years in error-related event-related potentials, which are thought to originate from preSMA/ACC (Miltner et al., 1997; Davies et al., 2004).

Together, these developmental trajectories suggest that children aged 8-9 learn from the informative value of feedback, with a focus toward valuing positive feedback. In contrast, 11- to 13year-olds learn from conflict signals that depend on a general performance-monitoring system. Finally, adults learn from both the informative value, with a focus toward evaluating negative feedback, and conflict signals. Some caution in the interpretation of these findings is warranted, because it depends on reverse inferences (Poldrack, 2006), but the findings highlight possible strategy differences across development in feedback-based learning. Moreover, previous behavioral modeling studies support the difference in informative value of negative feedback for adults versus child populations. A recent model states that children's networks show a decreased influence of negative reinforcement (e.g., negative feedback) relative to positive reinforcement compared with adult networks (Berkeljon and Raijmakers, 2007), consistent with the neural results shown in the current study. 
The results of this study fit well with previous developmental studies, which have demonstrated differential recruitment of prefrontal cortex and parietal cortex during cognitive control tasks (Klingberg et al., 2002; Crone et al., 2006a,b). Recently, researchers have shown that regions that are sensitive to reward and risks, in particular the orbitofrontal cortex and nucleus accumbens, are also sensitive to developmental change, in particular in adolescence (Ernst et al., 2005; Galvan et al., 2006). Interestingly, in these studies researchers demonstrated an early sensitivity to reward and an adult sensitivity to control demands. In this study, we did not observe developmental differences in emotion-related brain areas, but this may be the result of this task being a cognitive learning task, rather than an emotional risk-taking task. In future studies, it will be interesting to examine the developmental differences in feedback learning in this context as well (Eshel et al., 2007).

The current results show that the caudate, in contrast to preSMA/ACC, DLPFC, and superior parietal cortex, is sensitive to positive performance feedback, and this pattern was found for all age groups. These results are consistent with previous developmental imaging studies, which have reported early recruitment of the caudate in attention switching but late development of cortical areas (Casey et al., 2004). Thus, despite reports showing that the caudate has a slow structural developmental trajectory (Giedd, 2008), this study and others (Casey et al., 2004) report early functional involvement of the caudate. The results of the current study possibly indicate that brain areas that are responsive to positive performance feedback in adults function at adult level earlier than brain areas that are responsive to negative performance feedback in adults.

It should be noted that the caudate's response to positive feedback did not survive the significance threshold when additional analyses were performed that corrected for number of observations or movement differences. Apparently, its sensitivity to performance feedback is less robust than for the cortical areas. Indeed, a previous feedback-learning study failed to show a consistent effect of caudate in feedback processing (Zanolie et al., $2008 \mathrm{~b}$ ), and other studies suggest that subparts of the caudate are differentially activated for different phases of learning (Cincotta and Seger, 2007). Future studies are necessary to understand the caudate's role in feedback processing in more detail.

Two further caveats of this study should be noted. First, we did not find brain-behavior correlations. The absence of these correlations is possibly attributable to qualitative differences between age groups, with a shift in learning from positive to learning from negative feedback. Thus, the relation with behavior is most likely not linear. Second, in this study we directly compared learning from positive feedback with learning from negative feedback. In future studies, it will be important to compare these learning effects with a neutral baseline condition to investigate whether age groups differ mostly in learning from positive relative to neutral signals, learning from negative relative to neutral signals, or both.

\section{Conclusion}

The different neural sensitivity to the informative value of feedback across ages is striking. Whereas adults use control regions after signals of response adjustment, children show activation in most of these brain regions after signals of behavior continuation, with a transition around age 12 . In this study, the specific distinction between a narrow age range in development (8- and 12-yearolds) led to important insights in associated neural developmental changes. Additionally, even after age 12, important neural and behavioral changes occur in the feedback-based learning task. To our knowledge, this is the first feedback-learning study separating these age groups. This study also highlighted that different neural changes may be the result of strategy differences rather than a failure to recruit a certain brain area. These strategy differences, both between and within age groups, should be a hallmark for future studies trying to relate cognitive neuroscience to educational practice.

\section{References}

Barrouillet P, Lecas JF (1999) Mental models in conditional reasoning and working memory. Thinking and Reasoning 5:289-302.

Berkeljon A, Raijmakers MEJ (2007) An ART neural network model of discrimination learning. Paper presented at IEEE Sixth International Conference on Development and Learning, London, July.

Brass M, Derrfuss J, Forstmann B, von Cramon DY (2005) The role of the inferior frontal junction area in cognitive control. Trends Cogn Sci 9:314-316

Brett M, Anton JL, Valabregue R, Poline JB (2002) Region of interest analysis using an SPM toolbox. Paper presented at Eighth International Conference on Functional Mapping of the Human Brain, June, Sendai, Japan.

Brown JW, Braver TS (2005) Learned predictions of error likelihood in the anterior cingulate cortex. Science 307:1118-1121.

Bunge SA, Hazeltine E, Scanlon MD, Rosen AC, Gabrieli JD (2002) Dissociable contributions of prefrontal and parietal cortices to response selection. Neuroimage 17:1562-1571.

Casey BJ, Davidson MC, Hara Y, Thomas KM, Martinez A, Galvan A, Halperin JM, Rodríguez-Aranda CE, Tottenham N (2004) Early development of subcortical regions involved in non-cued attention switching. Dev Sci 7:534-542.

Cepeda NJ, Kramer AF, Gonzalez de Sather JC (2001) Changes in executive control across the life span: examination of task-switching performance. Dev Psychol 37:715-730.

Cincotta CM, Seger CA (2007) Dissociation between striatal regions while learning to categorize via feedback and via observation. J Cogn Neurosci 19:249-265.

Cocosco CA, Kollokian V, Kwan RK-S, Evans AC (1997) Brain web: Online interface to a 3D MRI simulated brain database. Neuroimage 5:S425.

Crone EA, Ridderinkhof KR, Worm M, Somsen RJ, van der Molen MW (2004) Switching between spatial stimulus-response mappings: a developmental study of cognitive flexibility. Dev Sci 7:443-455.

Crone EA, Wendelken C, Donohue S, van Leijenhorst L, Bunge SA (2006a) Neurocognitive development of the ability to manipulate information in working memory. Proc Natl Acad Sci U S A 103:9315-9320.

Crone EA, Donohue SE, Honomichl R, Wendelken C, Bunge SA (2006b) Brain regions mediating flexible rule use during development. J Neurosci 26:11239-11247.

Crone EA, Zanolie K, Van Leijenhorst L, Westenberg PM, Rombouts SA (2008) Neural mechanisms supporting flexible performance adjustment during development. Cogn Affect Behav Neurosci 8:165-177.

Dale AM (1999) Optimal experimental design for event-related fMRI. Hum Brain Mapp 8:109-114.

Davies PL, Segalowitz SJ, Gavin WJ (2004) Development of responsemonitoring ERPs in 7- to 25-year-olds. Dev Neuropsychol 25:355-376.

Ernst M, Nelson EE, Jazbec S, McClure EB, Monk CS, Leibenluft E, Blair J, Pine DS (2005) Amygdala and nucleus accumbens in responses to receipt and omission of gains in adults and adolescents. Neuroimage 25:1279-1291.

Eshel N, Nelson EE, Blair RJ, Pine DS, Ernst M (2007) Neural substrates of choice selection in adults and adolescents: development of the ventrolateral prefrontal and anterior cingulate cortices. Neuropsychologia $45: 1270-1279$

Galvan A, Hare TA, Parra CE, Penn J, Voss H, Glover G, Casey BJ (2006) Earlier development of the accumbens relative to orbitofrontal cortex might underlie risk-taking behavior in adolescents. J Neurosci 26:6885-6892.

Giedd JN (2008) The teen brain: insights from neuroimaging. J Adolesc Health 42:335-343.

Gogtay N, Giedd JN, Lusk L, Hayashi KM, Greenstein D, Vaituzis AC, Nugent TF 3rd, Herman DH, Clasen LS, Toga AW, Rapoport JL, Thompson PM 
(2004) Dynamic mapping of human cortical development during childhood through early adulthood. Proc Natl Acad Sci U S A 101:8174-8179.

Holroyd CB, Coles MG (2002) The neural basis of human error processing: reinforcement learning, dopamine, and the error-related negativity. Psychol Rev 109:679-709.

Huizinga M, Dolan CV, van der Molen MW (2006) Age-related change in executive function: developmental trends and a latent variable analysis. Neuropsychologia 44:2017-2036.

Kerns JG, Cohen JD, MacDonald AW 3rd, Cho RY, Stenger VA, Carter CS (2004) Anterior cingulate conflict monitoring and adjustments in control. Science 303:1023-1026.

Klingberg T, Forssberg H, Westerberg H (2002) Increased brain activity in frontal and parietal cortex underlies the development of visuospatial working memory capacity during childhood. J Cogn Neurosci 14:1-10.

Liston C, Matalon S, Hare TA, Davidson MC, Casey BJ (2006) Anterior cingulate and posterior parietal cortices are sensitive to dissociable forms of conflict in a task-switching paradigm. Neuron 50:643-653.

Mars RB, Coles MG, Grol MJ, Holroyd CB, Nieuwenhuis S, Hulstijn W, Toni I (2005) Neural dynamics of error processing in medial frontal cortex. Neuroimage 28:1007-1013.

Miller EK, Cohen JD (2001) An integrative theory of prefrontal cortex function. Annu Rev Neurosci 24:167-202.

Miltner WHR, Braun CH, Coles MGH (1997) Event-related brain potentials following incorrect feedback in a time-estimation task: evidence for a "generic" neural system for error detection. J Cogn Neurosci 9:788-798.

Poldrack RA (2006) Can cognitive processes be inferred from neuroimaging data? Trends Cogn Sci 10:59-63.

Qu L, Zelazo PD (2007) The facilitative effect of positive stimuli on 3-yearolds' flexible rule use. Cogn Dev 22:456-473.
Raven JC (1941) Standardization of progressive matrices. Br J Med Psychol 19:137-150.

Rushworth MF, Walton ME, Kennerley SW, Bannerman DM (2004) Action sets and decisions in the medial frontal cortex. Trends Cogn Sci 8:410-417.

Rushworth MFS, Buckley MJ, Behrens TEJ, Walton ME, Bannerman DM (2007) Functional organization of the medial frontal cortex. Curr Opin Neurobiol 17:220-227.

Schmittmann VD, Visser I, Raijmakers MEJ (2006) Multiple learning modes in the development of performance on a rule-based category learning task. Neuropsychologia 44:2079-2091.

Seger CA, Cincotta CM (2006) Dynamics of frontal, striatal, and hippocampal systems during rule learning. Cereb Cortex 16:1546-1555.

Talairach J, Tourneaux P (1988) Co-planar stereotaxic atlas of the human brain. Stuttgart, Germany: Thieme.

Toni I, Rowe J, Stephan KE, Passingham RE (2002) Changes of corticostriatal effective connectivity during visuomotor learning. Cereb Cortex 12:1040-1047.

Ullsperger M, von Cramon DY (2004) Decision making, performance and outcome monitoring in frontal cortical areas. Nat Neurosci 7:1173-1174.

Walton ME, Devlin JT, Rushworth MF (2004) Interactions between decision making and performance monitoring within prefrontal cortex. Nat Neurosci 7:1259-1265.

Zanolie K, Van Leijenhorst L, Rombouts SARB, Crone EA (2008a) Separable neural mechanisms contribute to feedback processing in a rulelearning task. Neuropsychologia 46:117-126.

Zanolie K, Teng S, Donohue SE, Van Duijvenvoorde ACK, Band GPH, Rombouts SARB, Crone EA (2008b) Intradimensional and extradimensional switching between colors and shapes on the basis of positive and negative feedback: an fMRI and EEG study on feedback based learning. Cortex 44:537-547. 